\title{
Numerical studies of the KP line-solitons
}

\author{
S. Chakravarty, T. McDowell, and M. Osborne \\ Department of Mathematics, University of Colorado, Colorado Springs, CO 80918, USA
}

\begin{abstract}
The Kadomtsev-Petviashvili (KP) equation admits a class of solitary wave solutions localized along distinct rays in the xy-plane, called the line-solitons, which describe the interaction of shallow water waves on a flat surface. These wave interactions have been observed on long, flat beaches, as well as have been recreated in laboratory experiments. In this paper, the line-solitons are investigated via direct numerical simulations of the the KP equation, and the interactions of the evolved solitary wave patterns are studied. The objective is to obtain greater insight into solitary wave interactions in shallow water and to determine the extent the KP equation is a good model in describing these nonlinear interactions.
\end{abstract}

Keywords: KP equation, line-solitons, numerical simulations, chord diagrams

\section{Introduction}

An important example of physically interesting nonlinear wave equations was proposed in 1970 by Kadomtsev and Petviashvili [2] in their study of plasma waves. It is a $(2+1)$-dimensional, weakly nonlinear dispersive wave equation of the form

$$
\left(4 u_{t}+6 u u_{x}+u_{x x x}\right)_{x}+3 u_{y y}=0,
$$

where $x, y, t$ are the spatial coordinates and time; $u=u(x, y, t)$ represents the (normalized) wave amplitude; the subscripts denote partial derivatives. Equation (1) is referred to as the KP equation throughout the text. We point out that (1) is the KP equation with negative dispersion, while the positive dispersion KP equation (not considered here) corresponds to (1) with a negative sign in front of the $u_{y y}$-term. The KP equation (1) is derived from the three-dimensional Euler equations for an irrotational and incompressible fluid under the assumptions that it describes the propagation of small amplitude, weakly dispersive, uni-directional waves with small, transverse variation. From a physical perspective, the KP equation has been studied in the context of oblique interactions of ion-acoustic and shallow water solitary waves. An example of such wave phenomena observed in nature is the surface wave patterns created by the oblique interaction of incoming waves in shallow water on long, flat beaches as shown in Figure 1.

The KP equation (1) admits an important class of solitary wave solutions that are regular, non-decaying, and localized along distinct lines in the $x y$-plane. These solutions are known as the line-soliton solutions, which in recent years, have been a subject of extensive research that led to a complete classification of these solutions using geometric and combinatorial techniques $[4,5,7]$. These solutions feature an arbitrary number of asymptotic line solitons in the far-field and a complex interaction pattern of intermediate

*Corresponding author. Email: chuck@ math.uccs.edu 

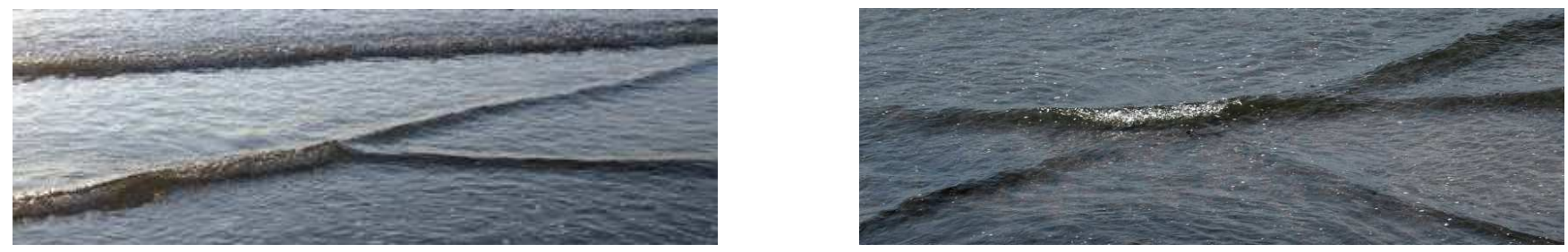

Figure 1: Beach wave patterns. Photographs by M. J. Ablowitz and D. E. Baldwin [3]

solitons resembling a web-like structure in the near-field region. Several examples of such exact solutions have been constructed in recent laboratory wave tank experiments demonstrating surface wave patterns generated by solitary wave interactions $[13,10]$.

It is important to consider the initial value problem of the KP equation in order to investigate twodimensional wave patterns generated by the interaction of initial solitary waves. However, at present, there is no available analytic method to study the KP initial value problem with non-decaying initial data in the form of solitary waves in the far-field region. In this paper, we investigate such initial value problems associated with the KP equation (1) numerically in order to gain insight into the interaction properties of the non-decaying solitary waves in shallow water. We perform direct numerical simulations of the KP equation with a variety of initial conditions, study the convergence of initial data to the exact solutions, and analyze the relation between the parameters defining the initial conditions and those of the exact solutions. We consider types of initial waves relevant to both physical problems and experiments. Finally, we compare the numerical simulations with the theoretical results derived for the KP line-solitons.

The paper is organized as follows: in section 2, we provide a brief summary of the theoretical development for the KP line-solitons and their classification obtained earlier in $[4,5]$. Section 3 describes the numerical scheme implemented in this paper and the proposed method to measure how close the numerical wave patterns are to the exact KP line-soliton solutions in a local $L^{2}$ sense. In section 4 , we describe our numerical results by considering some simple forms of piece-wise defined initial conditions. We take initial data consisting of two or more semi-infinite line solitons joined together, representing interacting solitary waves. Finally, in Section 5, we summarize our work and provide a brief outlook for future work.

\section{The KP line-solitons}

The simplest example of a KP line-soliton is the one-soliton solution, which is a traveling wave

$$
u(x, y, t)=\frac{1}{2}\left(k_{2}-k_{1}\right)^{2} \operatorname{sech}^{2} \frac{1}{2}\left(k_{2}-k_{1}\right)\left[x+\left(k_{1}+k_{2}\right) y-c_{12} t-x_{12}\right],
$$

and is localized along a line $L_{12}: x+\left(k_{1}+k_{2}\right) y-c_{12} t-x_{12}=0$ in the $x y$-plane for fixed $t$, as shown in Figure 2. The one-soliton is characterized by two real, distinct parameters $k_{1}<k_{2}$ which determine the soliton amplitude: $\frac{1}{2}\left(k_{2}-k_{1}\right)^{2}$, soliton speed: $c_{12}=k_{1}^{2}+k_{1} k_{2}+k_{2}^{2}$, and the soliton slope: $k_{1}+k_{2}=\tan \Psi_{12}$, where $\Psi_{12}$ is the angle, measured counterclockwise between the line $L$ and the positive $y$-axis. We usually denote a one-soliton solution as the $[1,2]$-soliton, since it is determined by the parameters $k_{1}$ and $k_{2}$.

The solution $u(x, y, t)$ of (1) is usually prescribed in terms of the $\tau$-function $\tau(x, y, t)$ as

$$
u(x, y, t)=2(\ln \tau)_{x x}
$$

For the general line-soliton solutions of KP, the $\tau$-function is a linear combination of exponential functions whose exponents are linear in $x, y$, and $t$. Furthermore, $\tau(x, y, t)$ depends on

(i) $M$ distinct real parameters $\left\{k_{1}, k_{2}, \ldots, k_{M}\right\}$ ordered as $k_{1}<k_{2}<\cdots<k_{M}$, and 

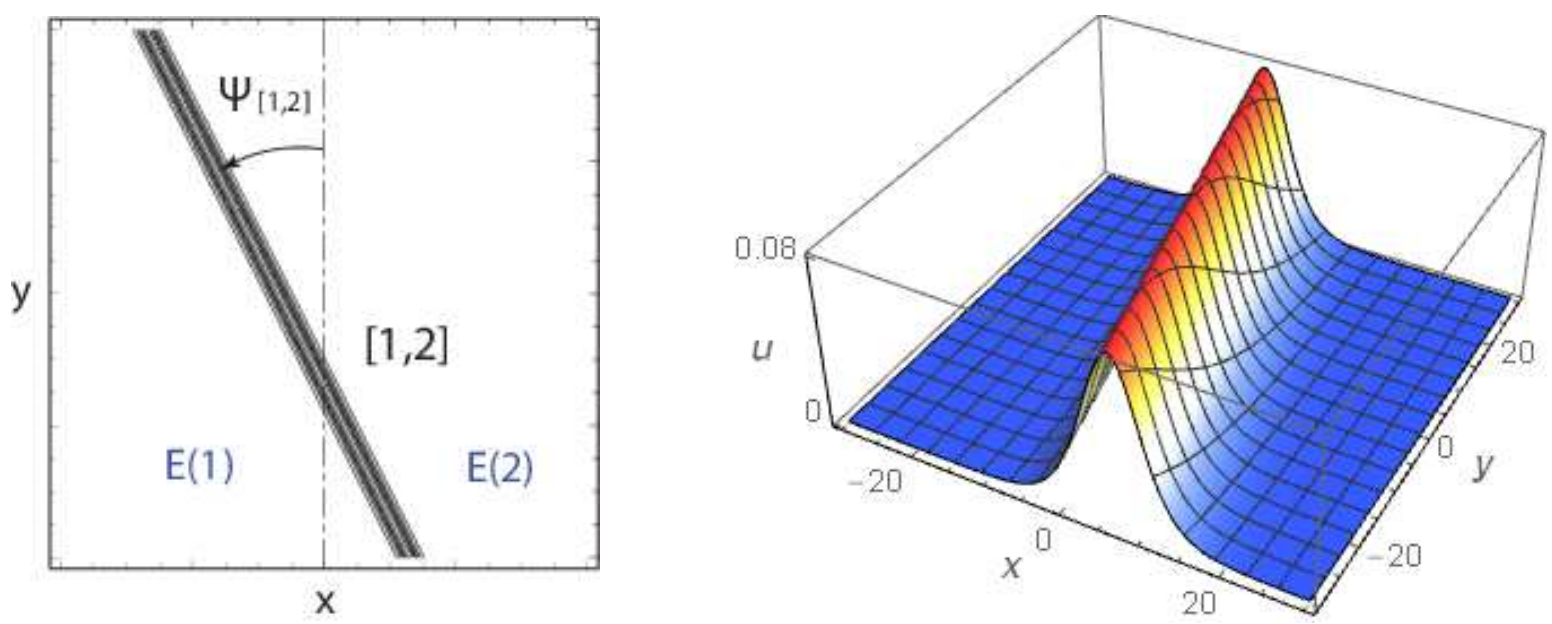

Figure 2: One-soliton solution of the KP equation

(ii) an $N \times M$ real matrix $A$ of full rank with $N<M$.

The explicit form of the $\tau$-function is the following $N \times N$ determinant

$$
\tau=\left|\left(\begin{array}{cccc}
a_{11} & \cdots & \cdots & a_{1 M} \\
a_{21} & \cdots & \cdots & a_{2 M} \\
\vdots & \vdots & & \vdots \\
a_{N 1} & \cdots & \cdots & a_{N M}
\end{array}\right)\left(\begin{array}{cccc}
E(1) & & & \\
& E(2) & & \\
& & \ddots & \\
& & & E(M)
\end{array}\right)\left(\begin{array}{cccc}
1 & k_{1} & \cdots & k_{1}^{N-1} \\
1 & k_{2} & \cdots & k_{2}^{N-1} \\
\vdots & \vdots & & \vdots \\
\vdots & \vdots & & \vdots \\
1 & k_{M} & \cdots & k_{M}^{N-1}
\end{array}\right)\right|,
$$

where $E(j):=\exp \left(k_{j} x+k_{j}^{2} y-k_{j}^{3} t\right)$ and $a_{i j}$ are the elements of the matrix $A$ mentioned above. The linesoliton solution $u(x, y, t)$ is non-singular for all $x, y$, and $t$ if and only if all principal minors $A\left(m_{1}, m_{2}, \ldots, m_{N}\right)$ of the matrix $A$, are non-negative [4,9]. Here, $A\left(m_{1}, m_{2}, \ldots, m_{N}\right)$ denotes the determinant of the $N \times N$ sub-matrix of $A$ with distinct columns labeled as $1 \leq m_{1}<m_{2}<\ldots<m_{N} \leq N$. Without loss of generality, the matrix $A$ can be taken in reduced row-echelon form.

For example, the $\tau$-function for the one-soliton solution (2) is given by $\tau(x, y, t)=E(1)+a E(2)$, where the positive parameter $a$ is related to the $x$-intercept of the line $L_{12}$ along which the soliton is localized by $x_{12}=\ln a$. This $\tau$-function is obtained by choosing $N=1, M=2$, and $A=(1, a)$ in (4). Note from Figure 2 that the one-soliton solution is exponentially small in regions of the $x y$-plane where the exponentials $E(1)$ and $E(2)$ are dominant, and is localized along the line $L_{12}$ where $E(1)$ and $E(2)$ are of the same order.

In previous works $[4,5,7]$, it was shown that the general soliton solution given by equations (3) and (4) consists of $N$ asymptotic line-solitons as $y \gg 0$ and $M-N$ asymptotic line-solitons as $y \ll 0$. This solution is referred to as a $(M-N, N)$-soliton solution. Each asymptotic line-soliton has the form of an exact 1-soliton solution (2), i.e., has the asymptotic form

$$
\frac{1}{2}\left(k_{j}-k_{i}\right)^{2} \operatorname{sech}^{2} \frac{1}{2}\left(k_{j}-k_{i}\right)\left[x+\left(k_{i}+k_{j}\right) y-c_{i j} t-x_{i j}\right],
$$

with $c_{i j}=k_{i}^{2}+k_{i} k_{j}+k_{j}^{2}$ and is uniquely parametrized by a pair of distinct $k$-parameters $k_{i}<k_{j}$ for $i<j$. Hence, we denote each asymptotic line-soliton as the $[i, j]$-soliton in this article. Furthermore, the index pair $[i, j]$ is uniquely characterized by a map $\pi$ such that $\pi(i)=j$ if $[i, j]$ labels an asymptotic soliton for $y \gg 0$, and $\pi(j)=i$ if $[i, j]$ labels an asymptotic soliton for $y \ll 0$. The map $\pi$ turns out to be fixed-point free permutation of the index set $[M]$ known as a derangement, which is conveniently represented by a chord diagram shown in the example below. Thus, each $(M-N, N)$-soliton solution (3) generated by the $\tau$-function (4) is represented by a chord diagram associated with the derangement $\pi$. 

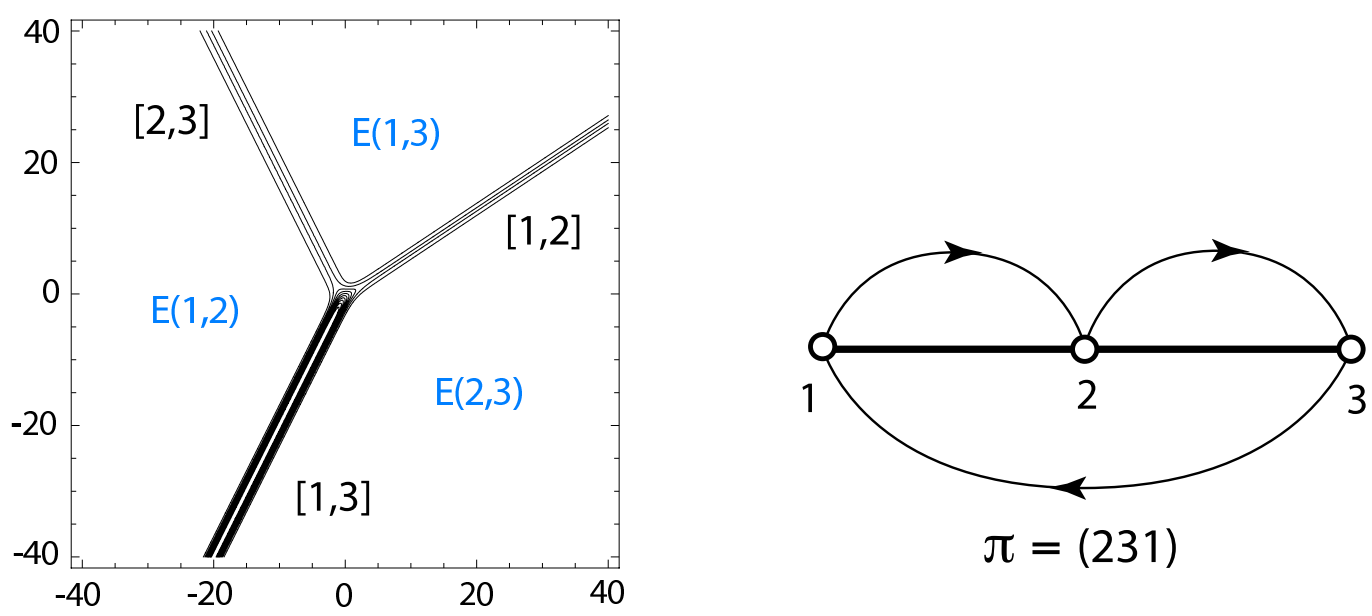

Figure 3: A $(1,2)$-soliton solution and its chord diagram. The $E(i, j)$ on each region indicates the dominant exponential term. The $k$-parameters are chosen as $\left(k_{1}, k_{2}, k_{3}\right)=\left(-\frac{5}{4},-\frac{1}{4}, \frac{3}{4}\right)$ and the parameters in the $A$ matrix are chosen as $a=\frac{1}{2}$ and $b=1$.

Example: The Y-soliton or (1,2)-soliton solution.

Let us consider the case $N=2, M=3$ in (4) with parameters $\left\{k_{1}, k_{2}, k_{3}\right\}$ and a $2 \times 3$ A-matrix of the form

$$
A=\left(\begin{array}{ccc}
1 & 0 & -b \\
0 & 1 & a
\end{array}\right), \quad a>0, b>0 .
$$

The corresponding $\tau$-function is given by

$$
\tau=\left(k_{2}-k_{1}\right) E(1,2)+a\left(k_{3}-k_{1}\right) E(1,3)+b\left(k_{3}-k_{2}\right) E(2,3),
$$

where $E(i, j)=E(i)+E(j)$. Figure 3 shows a contour plot of a Y-soliton obtained from (3) and the $\tau$-function in (6). The region of the $x y$-plane where each exponential function $E(i, j)$ dominates is also indicated in the figure. The asymptotic line solitons are localized along the boundary of such adjacent regions. That is, the dominant exponential functions balance one another along the boundary. For $y \gg 0$, the boundary between the regions with the dominant exponentials $E(1,2)$ and $E(1,3)$ defines the [2,3]soliton solution. This can be seen as follows: along this boundary the $\tau$-function can be approximated as $\tau \approx\left(k_{2}-k_{1}\right) E(1,2)+a\left(k_{3}-k_{1}\right) E(1,3)$ so that we have

$$
u=2(\ln \tau)_{x x} \approx u(x, y, t)=\frac{1}{2}\left(k_{3}-k_{2}\right)^{2} \operatorname{sech}^{2} \frac{1}{2}\left(k_{3}-k_{2}\right)\left[x+\left(k_{2}+k_{3}\right) y-c_{23} t-x_{23}\right]
$$

where $c_{23}$ and $x_{23}$ are given by

$$
c_{23}=k_{2}^{2}+k_{2} k_{3}+k_{3}^{2}, \quad x_{23}=\frac{1}{k_{3}-k_{2}}\left[\ln \frac{k_{2}-k_{1}}{k_{3}-k_{1}}-\ln a\right] .
$$

Similar analyses lead to the asymptotic [1,2]-soliton for $y \gg 0$ and the asymptotic $[1,3]$-soliton for $y \ll 0$ with $c_{i j}=k_{i}^{2}+k_{i} k_{j}+k_{j}^{2}$, and

$$
x_{12}=\frac{1}{k_{2}-k_{1}}\left[\ln \frac{k_{3}-k_{1}}{k_{3}-k_{2}}-\ln \frac{b}{a}\right], \quad x_{13}=\frac{1}{k_{3}-k_{1}}\left[\ln \frac{k_{2}-k_{1}}{k_{3}-k_{2}}-\ln b\right] .
$$

The map $\pi$ associated with the soliton indices is then obtained by observing that $\pi(1)=2, \pi(2)=3$, which are associated with the $[1,2]$ - and the $[2,3]$-solitons for $y \gg 0$. Likewise, $\pi(3)=1$, which is associated 
with the $[1,3]$-soliton for $y \ll 0$. This leads to the permutation $\pi=(231)$ of the indices $\{1,2,3\}$. The chord diagram in Figure 3 displays two upper chords representing the index mapping corresponding to the $[1,2]$ - and [2,3]-solitons for $y \gg 0$ and a lower chord mapping the indices of the $[1,3]$-soliton for $y \ll 0$. As mentioned earlier, the chord diagrams provide a convenient representation of the line-soliton solutions of KP and is a key idea that is used throughout this paper.

We remark that the Y-soliton solution is a resonant three-wave interaction of the asymptotic linesolitons $[1,2],[2,3]$, and $[1,3]$ at the vertex of the Y-junction. In this case, the wave-vector and frequency of each line-soliton are $\mathbf{K}_{i j}=\left(k_{j}-k_{i}, k_{j}^{2}-k_{i}^{2}\right), \Omega_{i j}=k_{j}^{3}-k_{i}^{3}$, and the resonant condition is given by

$$
\mathbf{K}_{13}=\mathbf{K}_{12}+\mathbf{K}_{23}, \quad \Omega_{13}=\Omega_{12}+\Omega_{23} .
$$

The resonant vertex $\left(x_{0}(t), y_{0}(t)\right)$ is the common intersection point of all three lines

$$
L_{i j}: x+\left(k_{i}+k_{j}\right) y=c_{i j} t+x_{i j}, \quad 1 \leq i<j \leq 3 .
$$

In fact, it suffices to take the intersection of any two of the three lines; the resonant condition ensures that the third line passes through the same point.

\section{Numerical simulations}

In this paper we carry out direct numerical simulations of the KP equation (1) in order to study the interaction properties of the line-solitons. Specifically, we show that the numerical solutions corresponding to certain types of initial conditions approach aymptotically in time to the exact solutions discussed in Section 2. We have developed MATLAB codes to run these numerical simulations with initial data that are non-decaying at the boundary of the numerical domain. The initial value problems considered here are essentially an infinite energy problem in the sense that the initial waves have support for $y \gg 0$ or $y \ll 0$ (or both), but their interactions take place in a finite region of the $x y$-plane. The accuracy of our numerical codes are tested by comparing the local (near the interaction region) $L^{2}$-estimates of the numerical solution with the exact line-soliton solution of the KP equation for various types of initial data.

Numerical Scheme: We solve the KP equation numerically using a pseudo-spectral scheme on a rectangular domain $D=\left\{(x, y):|x| \leq L_{x} / 2,|y| \leq L_{y} / 2\right\}$. We assume (see Remarks (i) and (ii) below) that the solution is periodic in both $x$ and $y$. It is then convenient to rescale $D$ to a fixed domain $D^{\prime}=\{(X, Y)$ : $|X| \leq \pi,|Y| \leq \pi\}$ by defining $X=\left(2 \pi / L_{x}\right) x$ and $Y=\left(2 \pi / L_{y}\right) y$ so that (1) becomes

$$
\left(u_{t}+P u u_{X}+Q u_{3 X}\right)_{X}+R u_{2 Y}=0, \quad P=\frac{3 \pi}{L_{x}}, Q=\frac{2 \pi^{3}}{L_{x}^{3}}, R=\frac{3 \pi L_{x}}{2 L_{y}^{2}} .
$$

Due to periodicity, we can express the solution as

$$
u(X, Y, t)=\sum_{l=-\infty}^{\infty} \sum_{m=-\infty}^{\infty} \hat{u}(l, m, t) e^{i(l X+m Y)}
$$

and reduce (7) into an ODE for the time evolution of the Fourier coefficients $\hat{u}(l, m, t)$, namely,

$$
\hat{u}_{t}+\frac{i l P}{2} \mathcal{N}(\hat{u})+i\left(\frac{R m^{2}}{l}-Q l^{3}\right) \hat{u}=0, \quad l \neq 0,
$$

where $\mathcal{N}(\hat{u})$ is the Fourier transform of $u^{2}$ which is numerically evaluated as $\mathcal{N}(\hat{u})=\operatorname{FFT}\left[(\operatorname{IFFT}(\hat{u})]^{2}\right)$. The ODE (8) can be expressed as

$$
\hat{v}_{t}+\alpha e^{c t} \mathcal{N}\left(\hat{v} e^{-c t}\right)=0
$$


where $c=i\left(R m^{2} / l-Q l^{3}\right), \hat{v}=\hat{u} e^{c t}, \alpha=i l P / 2$, and numerically solved using the Runge-Kutta (RK4) method for a given initial data $\hat{u}(l, m, 0)$ obtained from the Fourier Transform of $u(X, Y, 0)$. The solution $u(X, Y, t)$, and hence $u(x, y, t)$, is then reconstructed by taking the inverse Fourier Transform of $\hat{u}(l, m, t)$ for $l \neq 0$.

\section{Remarks:}

(i) When $l=0$, the ODE in (8) reduces to $\hat{u}(0, m, t)=0$ for all values of $m$ and $t$. This is called the zeromode condition, which is equivalent to the mean-free condition: $\int_{-\pi}^{\pi} u d X=0$ and must be satisfied for all periodic solutions. Our initial data (in the form of line-solitons) do not satisfy the mean-free condition, so we force the zero-modes $\hat{u}(0, m, t)$ to be zero in our numerical code. However, our numerical experiments suggest that the maximum error in the Fourier domain due to the violation of this condition remains small (see also [8]).

(ii) The pseudo-spectral scheme works well for vanishing boundary conditions in both $x$ and $y$ directions. However, the initial data used in our numerical simulations do not vanish at the boundaries of the numerical domain $D$. As a result, in our simulations, we get reflected waves from the boundary that affect the numerical domain. Furthermore, the amplitude of the wave near the boundary diminishes due to dispersive waves created at the boundary. To address these issues, we use a method developed by Tanaka [11] (see also [12]) to artificially modify the data near the boundary to maintain constant amplitude and unperturbed shape. For the types of initial data used in our numerical simulations, we modify the numerical solution by imposing zero boundary conditions in the $x$-direction and by "patching" with the exact one-soliton solutions in the $y$-direction near the boundary. Figure 4 below shows an example of a V-shape initial condition with the indicated patching region at the boundary.

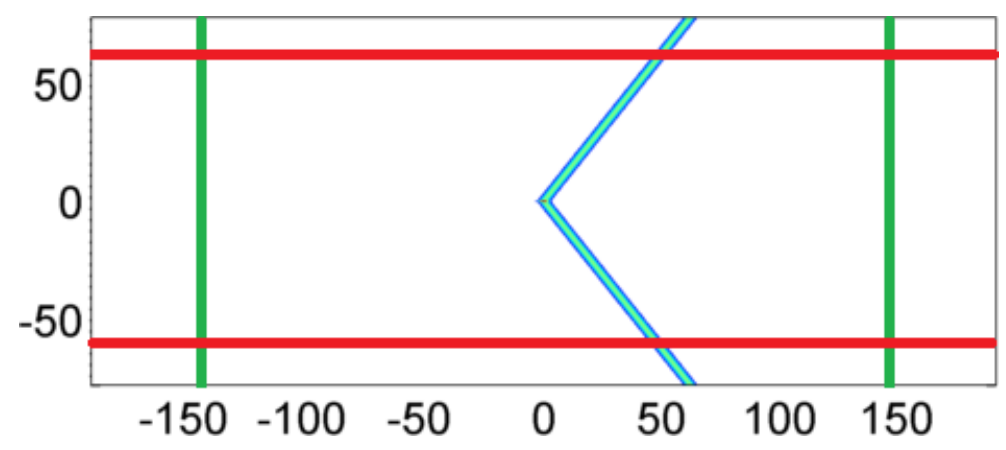

Figure 4: A V-shape initial condition with patching

We briefly outline below the procedure followed in our numerical simulations. We take a set of $k$ parameters to construct initial data obtained by gluing together two semi-infinite asymptotic line-solitons of the form given in (5) at $t=0$. This initial waveform corresponds to a "partial" chord diagram leading to several possible exact solutions. The numerical simulation then shows that such an initial condition evolves to an interacting wave-pattern which converges to an exact KP line-soliton solution. We clarify below what precisely we mean by convergence. First, we assume that the KP theory described in Section 2 holds for the evolving wave pattern. Hence, measuring the amplitudes $\frac{1}{2}\left(k_{j}-k_{i}\right)^{2}$ and slopes $k_{i}+k_{j}$ of the asymptotic line-solitons in this wave pattern allows us to identify each asymptotic line-soliton for $y \gg 0$ and $y \ll 0$. Using this information, we construct the derangement $\pi$ as explained in Section 2 and thus complete the partial chord diagram associated with the initial wave pattern. We remark here that in all of our numerical simulations, we find that the full chord diagram is obtained by the "minimal completion" of the partial chord diagram [7, 8]. Here, by minimal completion we mean that one adds extra chords so that the complete chord diagram for the permutation $\pi$ has the minimum total length (see examples in Section 
4). Using combinatorial techniques whose descriptions are beyond the scope of this paper, it is possible to recover the structure of the $A$-matrix associated with the exact KP line-soliton solution from the chord diagram associated with a given wave pattern $[4,9]$. Note however, that this theoretical result gives only the form of the $A$-matrix in its reduced row-echelon form, but not the exact value of its entries.

Next, we define a local error function as follows:

$$
E(t)=\left[\frac{\iint_{B}\left|u_{\text {num }}(x, y, t)-u_{\text {exact }}(x, y, t)\right|^{2} d x d y}{\iint_{B} u_{\text {exact }}^{2}(x, y, t) d x d y}\right]^{\frac{1}{2}} .
$$

The $L^{2}$ norms are computed over a rectangular box

$$
B:=\left\{(x, y) \in \mathbb{R}^{2}:\left|x-x_{0}(t)\right| \leq l_{x},\left|y-y_{0}(t)\right| \leq l_{y}\right\}
$$

where $\left(x_{0}(t), y_{0}(t)\right)$ is chosen to be an intersection point of two interacting line-solitons, and $l_{x}, l_{y}$ are chosen to be sufficiently large such that the the moving rectangle $B$ encloses the interaction region in the $x y$-plane for all $t>0$ during the simulation. The time-dependence of the interaction vertex $\left(x_{0}(t), y_{0}(t)\right)$ is determined from the exact KP solution. For instance, in the Y-soliton example in Section 2, the point where all three line-solitons intersect is taken to be the interaction vertex. We then construct the $A$-matrix by choosing its undetermined entries such that the error function $E(t)$ is minimized at a fixed time $t=T_{0}$. Note that this yields an exact KP line-soliton solution which is closest to the numerically computed waveform on the rectangle $B$ at $t=T_{0}$ in a local $L^{2}$-sense. We then confirm that the error, $E(t)$, decreases for $t>T_{0}$ with the same exact solution (i.e., whose $A$-matrix entries were optimized at $t=T_{0}$ ) up to sufficiently large time before the moving box $B$ gets too close to the patching region near the boundary of the numerical domain.

\section{Numerical results}

In this section, we present the results of our numerical simulations, which show the evolution of various types of solitary wave initial data to an exact solution of the KP equation. A typical initial data is formed by gluing together pieces of $[i, j]$ one-soliton solutions given by (5) for different values of the parameters $k_{i}$ and $k_{j}$. In our numerical simulations, we take the entire numerical domain to be $[-192,192]$ in $x$ and $[-78,78]$ in $y$. The unpatched region (see Remark (ii), Section 3) is $[-144,144]$ in $x$ and $[-58.5,58.5]$ in $y$. In order to strike a balance between accuracy and speed, we settle for 8 Fourier modes per unit length in both $x$ and $y$ directions for the FFT calculations. The spatial error is within $10^{-10}$ for all our simulations. We choose a time step of $\Delta t=0.005$ for the Runge-Kutta scheme. This keeps our error within $2.5 \times 10^{-2}$ for a typical run up to $t=40$.

\subsection{Y-soliton}

The Y-soliton solution was described earlier as an example in Section 2. It represents a resonant interaction of 3 one-soliton solutions. In our numerical experiments, we take the initial data consisting of a $[1,3]$ and $[1,2]$ soliton glued together at $(x, y)=(0,0)$, as shown on the left frame of the top panel in Figure 5 . Specifically, the initial condition is

$$
u(x, y, 0)= \begin{cases}\frac{1}{2}\left(k_{2}-k_{1}\right)^{2} \operatorname{sech}^{2} \frac{1}{2}\left(k_{2}-k_{1}\right)\left[x+\left(k_{1}+k_{2}\right) y\right], & y \geq 0 \\ \frac{1}{2}\left(k_{3}-k_{1}\right)^{2} \operatorname{sech}^{2} \frac{1}{2}\left(k_{3}-k_{1}\right)\left[x+\left(k_{1}+k_{3}\right) y\right], & y \leq 0\end{cases}
$$



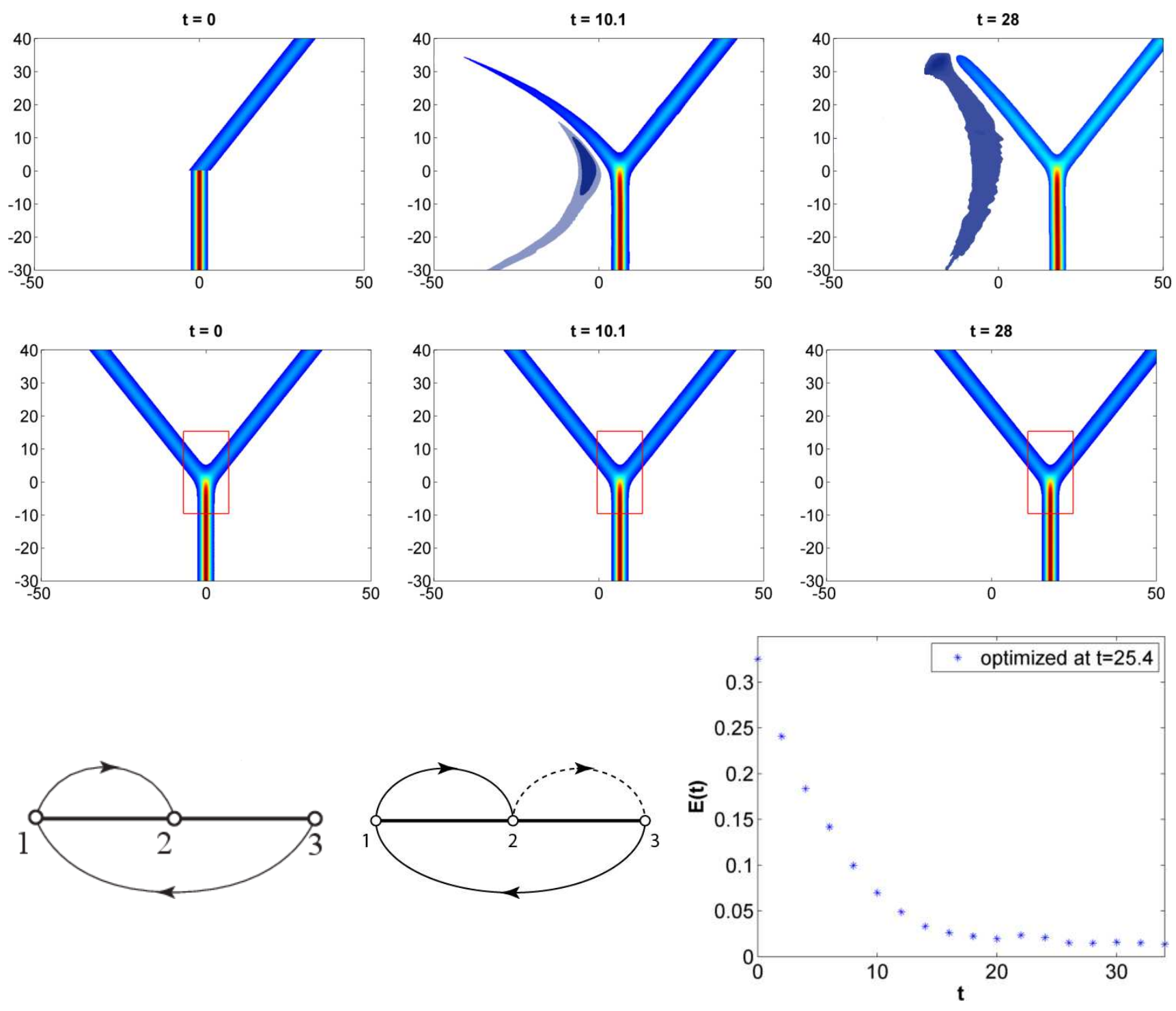

Figure 5: Numerical simulation of a Y-soliton. Top panel: numerical simulation of the initial data (left frame). Middle panel: Time evolution of the exact solution with optimized parameters. Bottom panel: The incomplete chord diagram and its completion (left and middle frames). Error $E(t)$ vs $t$ minimized at $t=25.4$ (right frame).

The $k$-parameters are chosen to be $\left\{k_{1}, k_{2}, k_{3}\right\}=\{-0.8,0,0.8\}$. The middle frame (top panel) of Figure 5 shows the formation of the $[2,3]$ soliton as well as dispersive waves created due to the resonant interaction of the initial waves at the intersection point (junction of the Y-shape). Note that since $c_{i j}=\left(k_{i}^{2}+k_{i} k_{j}+k_{j}^{2}\right)>0$, the $x$-component of the velocity of the $[i, j]$-soliton is always positive. That is, a line-soliton propagates in the positive $x$-direction. On the other hand, dispersive waves propagate in the negative $x$-direction. This can be seen from the dispersion relation of the KP equation (1) for a linear wave $\phi=\exp (i \mathbf{k} \cdot \mathbf{x}-i \omega t)$ with wave-vector $\mathbf{k}=\left(k_{x}, k_{y}\right)$ and frequency $\omega=\left(3 k_{y}^{2}-k_{x}^{4}\right) / 4 k_{x}$. The group velocity of the dispersive wave is then given by

$$
\mathbf{v}=\left(\frac{\partial \omega}{\partial k_{x}}, \frac{\partial \omega}{\partial k_{y}}\right)=\left(-\frac{3}{4}\left(k_{x}^{2}+\frac{k_{y}^{2}}{k_{x}^{2}}\right), \frac{3}{2} \frac{k_{y}}{k_{x}}\right) .
$$

Thus, the line-soliton solution separates from small radiations asymptotically in time, similar to the case of the KdV equation (see for example [1]). In our numerical simulations, we observe that the numerical Ysoliton moves to the right and eventually separates from the dispersive waves as shown in the right frame of 
the top panel of Figure 5. The first two figures in the bottom panel of Figure 5 shows the incomplete chord diagram (left frame) corresponding to the initial data and the complete chord diagram (middle frame) corresponding to the Y-soliton.

Next, we demonstrate numerically that the initial waves converge to the exact solution of the KP equation in the local sense as described at the end of Section 3. The exact Y-soliton solution is given by (3) and (6). By measuring the amplitude and the slope of the asymptotic line-solitons of the numerical solution, we find that the $k$-parameters are almost exactly the same (the maximum error is less than $10^{-4}$ ) as the initial values $\left\{k_{1}, k_{2}, k_{3}\right\}=\{-0.8,0,0.8\}$. We optimize the parameters $a$ and $b$ in the $A$-matrix so that the error in (9) is minimum at $t=25.4$ inside a rectangular box $B$ with $l_{x}=14$ and $l_{y}=25$ shown in the middle panel of Figure 5. The optimized value of the parameters are $(a, b)=(0.6,1.17)$, and the $A$-matrix is given by

$$
A=\left(\begin{array}{ccc}
1 & 0 & -1.17 \\
0 & 1 & 0.6
\end{array}\right)
$$

As explained in Section 3, this minimization scheme identifies an exact Y-soliton solution with the above $k$-values and $A$-matrix that is closest to the numerical solution. The middle panel in Figure 5 shows the evolution of the constructed exact solution with the optimized parameters and the rectangular box $\mathrm{B}$ enclosing the interaction region. Here, the center of the box $B$ is taken as the common intersection point of the three resonantly interacting line-solitons (see Example in Section 2) and can be computed from the exact Y-soliton solution. The center is given by $x_{0}(t)=0.64 t-0.098$ and $y_{0}(t)=-0.16$, which depends on the $k$-parameters and the optimized value of the parameters $(a, b)$. We compute the error, $E(t)$, for larger times until $t=35$ and observe that the error decreases as shown in the rightmost frame in the bottom panel of Figure 5.

Remark: Instead of varying the parameters $(a, b)$ of the exact solution during the optimization process, it is convenient to vary the soliton parameters $x_{i j}$, representing the $x$-intercepts of the $[i, j]$-solitons at $t=0$. It should be clear from the expressions for the $x_{i j}$ in the Y-soliton example of Section 2 that optimizing any two of the three $x$-intercepts, for example, $x_{13}$ and $x_{12}$, for fixed values of the $k$-parameters is equivalent to the optimization of the parameters $(a, b)$. Furthermore, a small change in $x_{i j}$ causes an exponentially large change in $(a, b)$. Therefore, a higher accuracy in the optimization process can be achieved by varying the $x_{i j}$ instead. In our simulations, we chose $x_{12}$ and $x_{13}$ as our optimization parameters. The optimized values were $\left(x_{12}, x_{13}\right)=(0.029,-0.098)$. Note, however, that in the initial condition, $x_{12}=x_{13}=0$ since the initial $[1,2]$ - and $[1,3]$-solitons meet at the origin. This implies that the asymptotic line-solitons in the numerical solution experience a shift due to their interaction with the dispersive waves. It can be easily verified from the expressions for the shifts $x_{i j}$ given in the Example of Section 2 that $\left(k_{2}-k_{1}\right) x_{12}+\left(k_{3}-k_{2}\right) x_{23}=\left(k_{3}-k_{1}\right) x_{13}$. Hence, it follows that the $[2,3]$-soliton experiences a relatively large negative shift. This is due to the fact that the $[2,3]$-soliton has a small amplitude and propagates with a slower speed immediately after its formation from the interaction of the [1,2]- and [1,3]solitons. This causes a negative shift of the [2,3]-soliton, while the negative shift of the $[1,3]$-soliton is because it loses momentum due to generation of the $[2,3]$-soliton and dispersive waves due to the nonlinear interaction.

\subsection{O-type soliton}

In this example, we consider initial data which is symmetric with respect to the $x$-axis and is obtained by gluing two semi-infinite line solitons in the form of a $<$-shape (or sideways V-shape) at the origin (see left frame in the top panel of Figure 7 below). Specifically, we choose

$$
u(x, y, 0)= \begin{cases}\frac{1}{2}\left(k_{2}-k_{1}\right)^{2} \operatorname{sech}^{2} \frac{1}{2}\left(k_{2}-k_{1}\right)\left[x+\left(k_{1}+k_{2}\right) y\right], & y \geq 0, \\ \frac{1}{2}\left(k_{4}-k_{3}\right)^{2} \operatorname{sech}^{2} \frac{1}{2}\left(k_{4}-k_{3}\right)\left[x+\left(k_{3}+k_{4}\right) y\right], & y \leq 0,\end{cases}
$$


which corresponds to a [1,2]- and [3,4]-soliton glued together in the right half of the $x y$-plane. The four
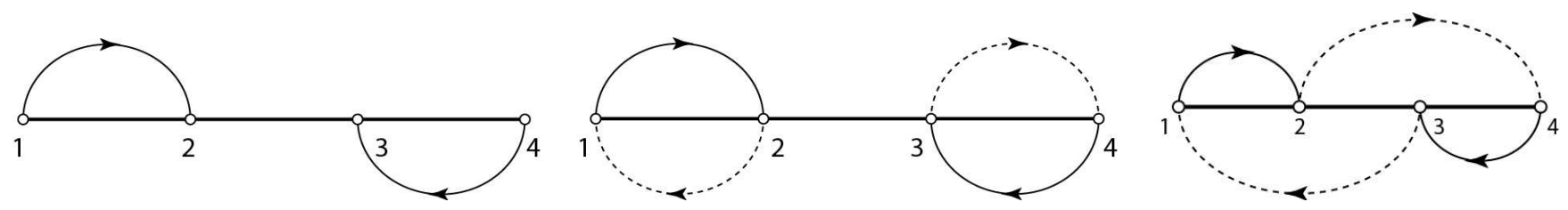

Figure 6: Possible chord diagrams for O-type initial data

$k$-parameters are chosen to be $\left\{k_{1}, k_{2}, k_{3}, k_{4}\right\}=\{-1.1,-0.1,0.1,1.1\}$, which are symmetric about $k=0$. Consequently, the [1,2]- and [3,4]-solitons have the same amplitude and opposite slopes, i.e., $\Psi_{12}=-\Psi_{34}$. The incomplete chord diagram corresponding to the initial condition is shown in the first frame of Figure 6. In this case there are two possible ways to complete the diagram. One will yield the chord diagram corresponding to the permutations $\pi=(2143)$ while the other will correspond to $\pi=(2413)$, as shown in the second and third frames in Figure 6. However, the minimal completion theory mentioned toward the end of Section 3 postulates that the initial data evolves to an exact KP (2,2)-soliton solution that corresponds to the middle chord diagram $(\pi=(2143))$ in Figure 6 . This particular solution is often referred to as the O-type soliton [6,4] which consists of a pair of [1,2]- and [3,4]-solitons for both $y \gg 0$ and $y \ll 0$, forming an X-shape. Our numerical simulations presented in Figure 7 demonstrate the formation of an Otype $(2,2)$-soliton solution, confirming the minimal completion theory. The top panel in Figure 7 shows the evolution of the <-shape initial data. The initial [1,2]- and [3,4]-half-solitons in $x \geq 0$ (right half plane) interact to create a pair of [1,2]- and [3,4]-half-solitons in $x<0$ together with dispersive waves. We

confirm this numerically by measuring the amplitudes $\frac{1}{2}\left(k_{j}-k_{i}\right)^{2}$ and the slopes $k_{i}+k_{j}$ of the asymptotic line-solitons in the wave pattern. The dispersive waves separate from the X-shape soliton wave pattern, which propagates in the positive $x$-direction as $t$ increases.

Next, we construct the exact solution from the O-type chord diagram shown in the middle frame of Figure 6 . The $A$-matrix and the $\tau$-function for the O-type soliton are given by $[4,5]$

$A=\left(\begin{array}{llll}1 & a & 0 & 0 \\ 0 & 0 & 1 & b\end{array}\right), \quad \tau=\left(k_{3}-k_{1}\right) E(1,3)+a\left(k_{3}-k_{2}\right) E(2,3)+b\left(k_{4}-k_{1}\right) E(1,4)+a b\left(k_{4}-k_{2}\right) E(2,4)$,

where $a>0, b>0$, and as before, $E(i, j)=E(i)+E(j)$ with $E(i)=\exp \left(k_{i} x+k_{i}^{2} y-k_{i}^{3} t\right)$. By minimizing the error function, $E(t)$, at $t=34.4$, we find the optimized values of the parameters $(a, b)=$ $(1.833,0.167)$. The middle panel in Figure 7 shows the evolution of the exact solution with these $(a, b)$ and $\left\{k_{1}, k_{2}, k_{3}, k_{4}\right\}=\{-1.1,-0.1,0.1,1.1\}$. The box size for this numerical simulation is $25 \times 35$, and the center of the box, chosen to be the intersection point of the [1,2]- and [3,4]-half-solitons in $x>0$, is given by $x_{0}(t)=1.33 t$ and $y_{0}(t)=0$. The bottom panel in Figure 7 shows the error $E(t)$ which decreases monotonically in time.

\subsection{2-soliton}

We consider another example of the symmetric, <-shape initial data, which evolves to a different $(2,2)$ soliton solution instead of the O-type soliton. In this case, the initial condition is

$$
u(x, y, 0)= \begin{cases}\frac{1}{2}\left(k_{3}-k_{1}\right)^{2} \operatorname{sech}^{2} \frac{1}{2}\left(k_{3}-k_{1}\right)\left[x+\left(k_{1}+k_{3}\right) y\right], & y \geq 0, \\ \frac{1}{2}\left(k_{4}-k_{2}\right)^{2} \operatorname{sech}^{2} \frac{1}{2}\left(k_{4}-k_{2}\right)\left[x+\left(k_{2}+k_{4}\right) y\right], & y \leq 0 .\end{cases}
$$

The left frame of the top panel in Figure 8 shows the <-shape initial condition formed by gluing together two one-soliton solutions with $k$-parameters $\left\{k_{1}, k_{2}, k_{3}, k_{4}\right\}=\{-0.9,-0.1,0.1,0.9\}$. The top and bottom 

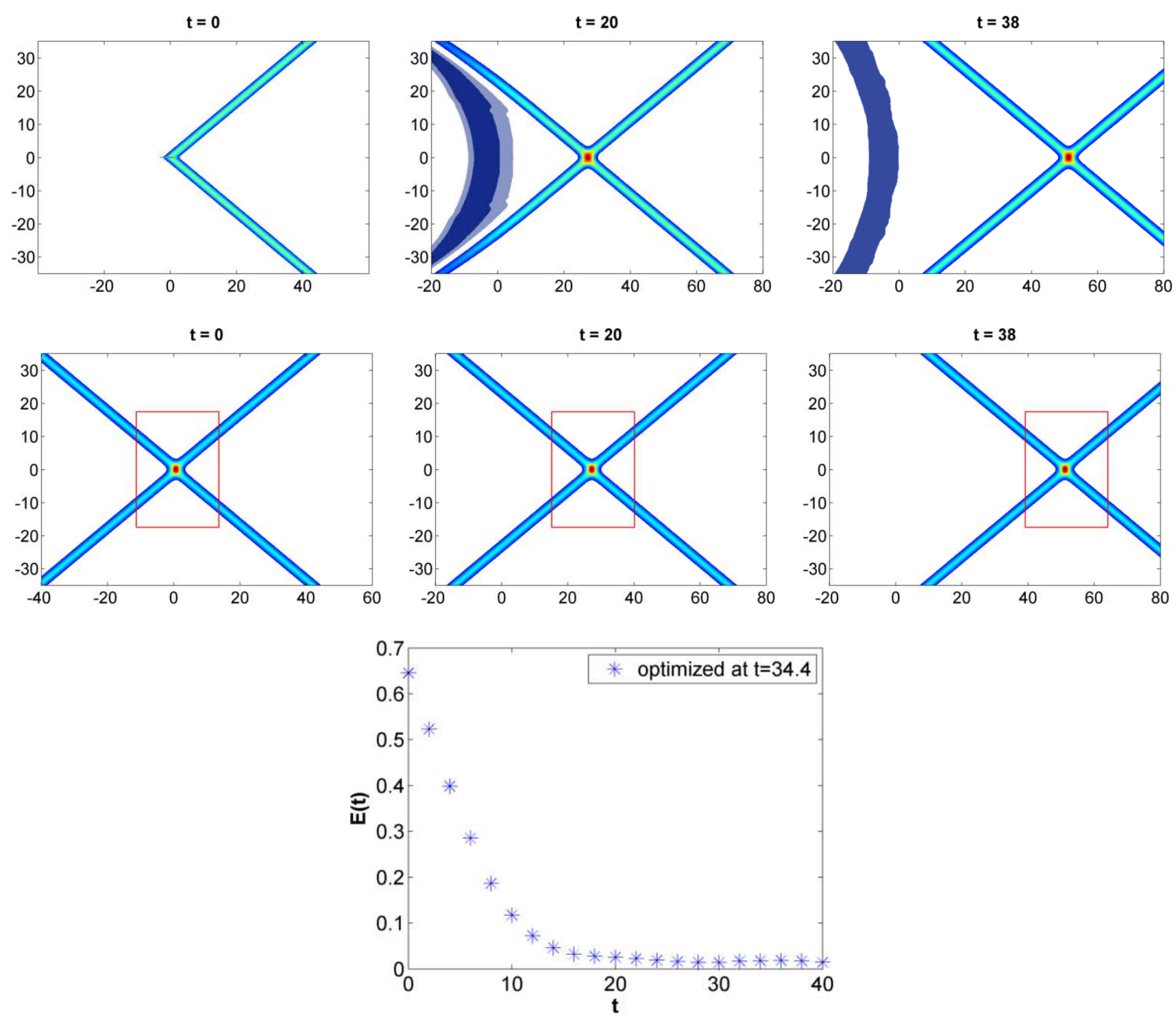

Figure 7: Simulation of an O-type soliton. Top panel: Numerical evolution of a <-shape initial data. Middle panel: Evolution of an exact O-soliton with optimized parameters. Bottom panel: Error curve, $E(t)$ minimized at $t=34.4$.

solitons of the <-shape are $[1,3]$ and $[2,4]$ solitons, respectively. The next two frames in the top panel show the time-evolution of the the initial data to a $(2,2)$-soliton wave pattern together with dispersive waves, which separate from the solitons. This pattern consists of $[1,3]$ - and $[3,4]$-solitons for $y>0$, $[1,2]$ - and $[2,4]$-solitons for $y<0$, and an intermediate $[1,4]$-soliton in the form of a vertical stem which interacts resonantly with both soliton pairs for $y \gg 0$ and $y \ll 0$. Thus, this solution can be viewed as a Yand a $X$-soliton glued together by the $[1,4]$-stem. The chord diagram for this solution corresponds to the permutation $\pi=(3142)$, hence we call it the 3142-soliton. Note that this chord diagram is the minimal completion of the incomplete chord diagram corresponding to the initial data. Both chord diagrams are shown in the first two frames in the bottom panel of Figure 8.

The $\tau$-function for the exact 3142-soliton depends on $k_{i}$ given above, and three other positive parameters $a, b$, and $c$ appearing in the $A$-matrix. That is,

$$
A=\left(\begin{array}{cccc}
1 & a & 0 & -b \\
0 & 0 & 1 & c
\end{array}\right), \quad \tau=\sum_{1 \leq i<j \leq 4} A(i j)\left(k_{j}-k_{i}\right) E(i, j),
$$



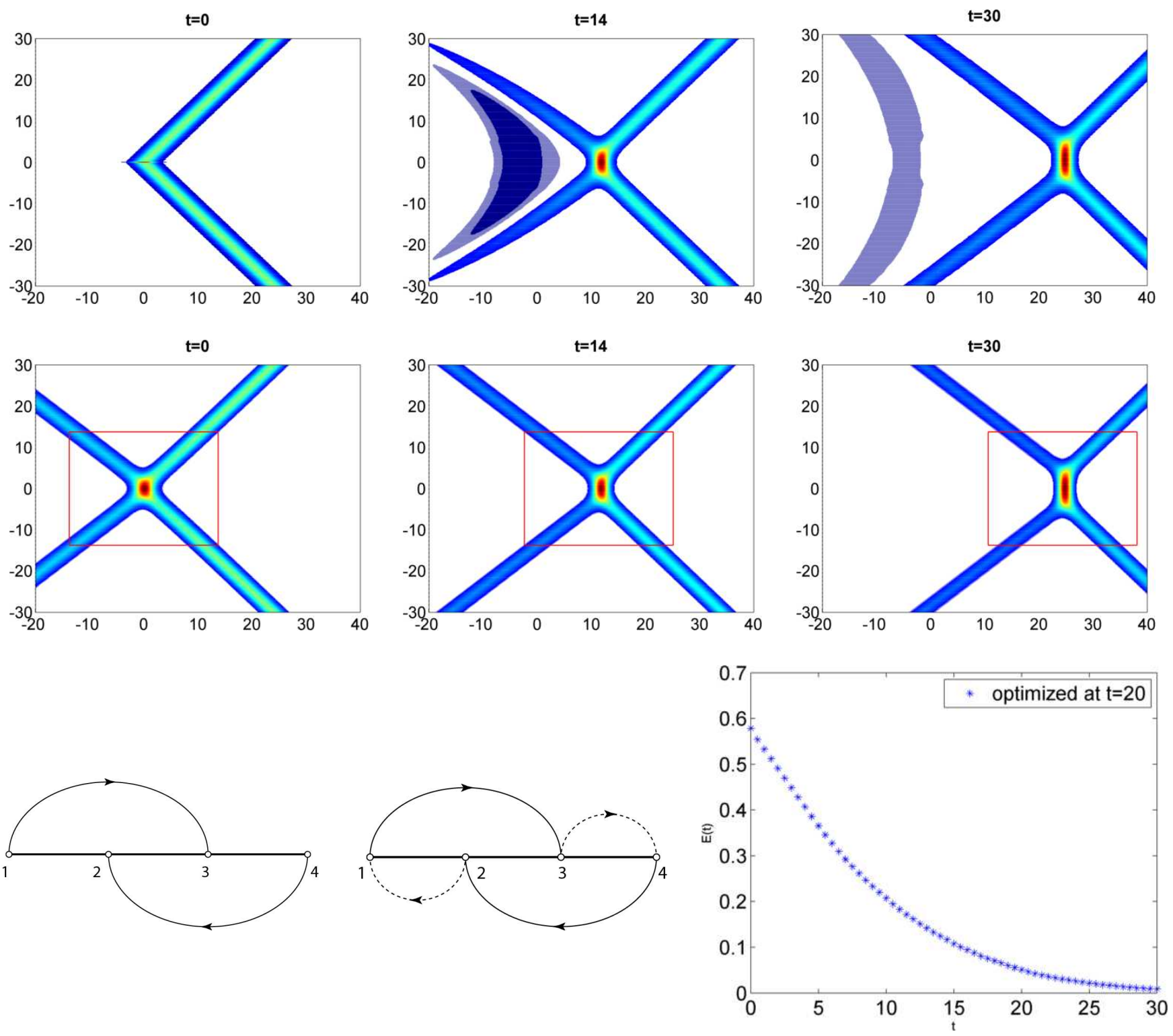

Figure 8: Simulation of a 3142-soliton. Top panel: numerical evolution of a $<$-shape initial data. Middle panel: evolution of the exact solution. Bottom panel: incomplete chord diagram for the initial data and its completion to $\pi=(3142)$ (left and middle frames). The error curve $E(t)$ vs $t$ is shown in the right frame.

where the principal minors are $A(12)=0, A(13)=1, A(14)=c, A(23)=a, A(34)=b, A(24)=a c$. Like the previous examples, we optimize the parameters $a, b$, and $c$ so that the error defined by equation (9) is minimized. This optimization is done by varying the $x$-intercepts $x_{i j}$ of the initial solitons $[1,3],[2,4]$, and the $[1,4]$-soliton. In much of the same way as in the Y-soliton example of Section 2 (see also Remark at the end of Section 4.1), these $x$-intercepts are related to the $A$-matrix parameters via the following relations $[5,8]$

$$
s=e^{\left(k_{1}-k_{4}\right) x_{14}}, \quad a=\frac{k_{3}-k_{1}}{k_{3}-k_{2}} s e^{\left(k_{4}-k_{2}\right) x_{24}}, \quad b=\frac{k_{3}-k_{1}}{k_{4}-k_{3}} s, \quad c=\frac{k_{3}-k_{1}}{k_{4}-k_{1}} s e^{\left(k_{3}-k_{1}\right) x_{13}} .
$$

For this numerical experiment, the optimal parameter values determining the exact (3142)-solution were computed at $t=20$ and are given by $x_{14}=0.476$ and $x_{13}=x_{24}=0.006$. From the initial condition $u(x, y, 0)$ one should expect that $x_{13}=x_{24}=0$; however, a small positive value of those $x$-intercepts in the exact solution suggests that the $[1,3]$ - and $[2,4]$-soliton gains a positive momentum (hence a positive 
$x$-shift) due to the creation of a high amplitude [1,4]-soliton or stem. Note that for the exact solution, the stem is already formed at $t=0$, as seen in the left frame of the middle panel of Figure 8 . This fact also accounts for the positive value for $x_{14}$. The optimized values of the $x_{i j}$ above correspond to the values $(a, b, c)=(2.135,0.531,0.237)$ for the parameters in the $A$-matrix. The exact 3142-soliton with these parameters are plotted in the middle panel of Figure 8 . The strictly monotonic decrease of the error, $E(t)$, shown in the bottom right panel of Figure 8 indicates that the numerical solution is converging to the exact (3142)-solution. Here, $E(t)$ is computed over a box of size $27.5 \times 27.5$, and the center of the box $\left(x_{0}(t), y_{0}(t)\right)=(0.81 t+0.476,0)$ is taken to be the intersection of the $[1,4]$-soliton with the $x$-axis.

Stem of the 3142-soliton: The stem soliton is formed by the resonant interactions of the $[1,3]-,[3,4]-$ solitons for $y>0$, and the $[1,2]-,[2,4]-$ solitons for $y<0$. It is the middle (high intensity) region visible in the top and middle panels of Figure 8. According to KP theory, the stem is an intermediate
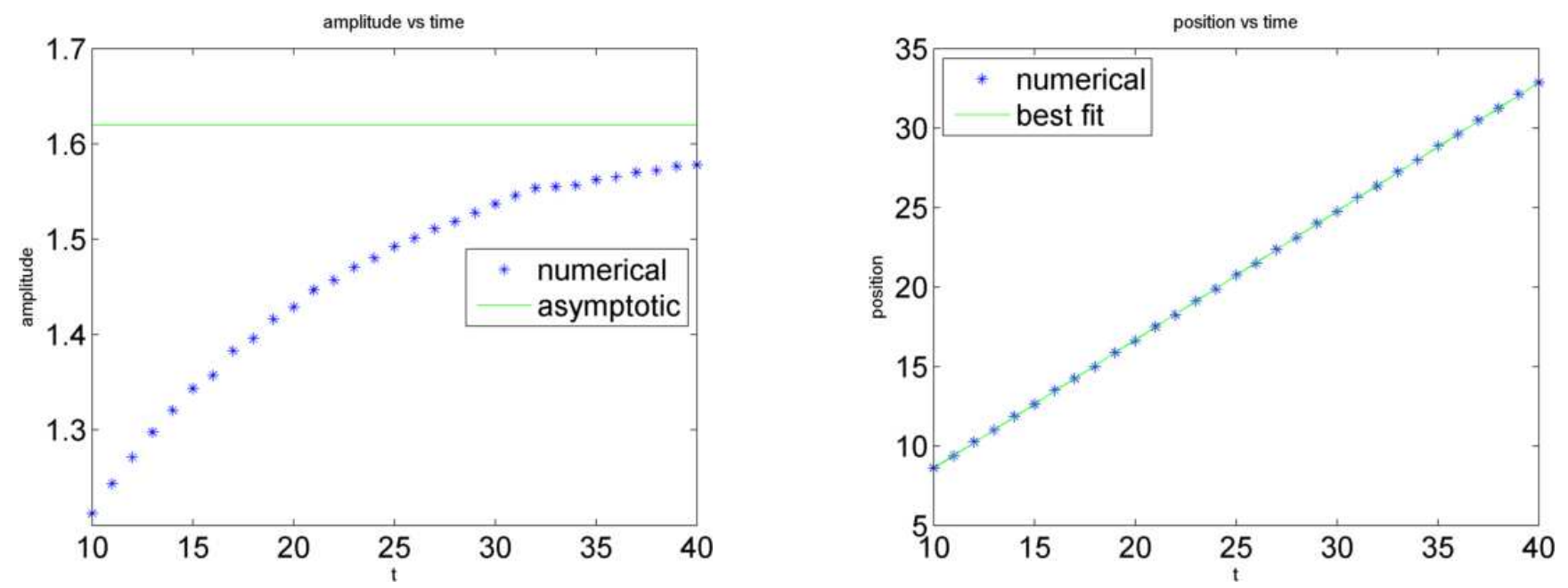

Figure 9: Stem amplitude \& position for the 3142-soliton

$[1,4]$-soliton which depends on the parameters $k_{1}$ and $k_{4}$. From (5), the amplitude and velocity of the stem are $\frac{1}{2}\left(k_{4}-k_{1}\right)^{2}$ and $c_{14}=k_{1}^{2}+k_{1} k_{4}+k_{4}^{2}$, respectively. We estimate both amplitude and velocity of the stem from the numerical solution and compare them with the theoretical values. The left frame in Figure 9 is a plot of the stem amplitude measured at $y=0$ with time. As the stem develops, its amplitude grows and gets closer asymptotically (from below) to the theoretical value $\frac{1}{2}\left(k_{4}-k_{1}\right)^{2}=1.62$, as shown by the solid line in the graph. The growth rate of the amplitude is slow, and the numerically estimated value stays below the asymptotic value for simulation time up to $t=40$. Our run time is limited by the boundary effects as the numerical wave form propagates too far right near the patching region. The right frame of Figure 9 shows the location of the point on the stem at $y=0$ as it evolves in time. The graph is linear and its slope yields a numerical estimate of the stem speed. For this simulation, the numerically estimated stem speed $=0.809$, which is in agreement with the theoretical value of $k_{1}^{2}+k_{1} k_{4}+k_{4}^{2}=0.81$ with $\left\{k_{1}, k_{2}, k_{3}, k_{4}\right\}=\{-0.9,-0.1,0.1,0.9\}$. It should be clear from Figure 8 that the stem evolves to an intermediate soliton whose length increases with time. The theoretical estimate of the stem length is given by $L(t)=2 k_{3} t+L_{0}$, where $L_{0}$ is a constant that depends on the parameters $a, b, c$, and the $k_{i}$. We compare the stem length of the numerical solution with the theoretical estimate in Figure 10. After adjusting for the soliton width, the theoretical line (slope $=2 k_{3}=0.2$ ) is a little lower than the best fit line (slope $=0.208$ ) for the numerical data. However, both lines have almost the same slope. Note that we have plotted the stem length from $t=10$ instead of $t=0$ because it takes some time (for the stem to grow sufficiently large) before it can be measured accurately. 


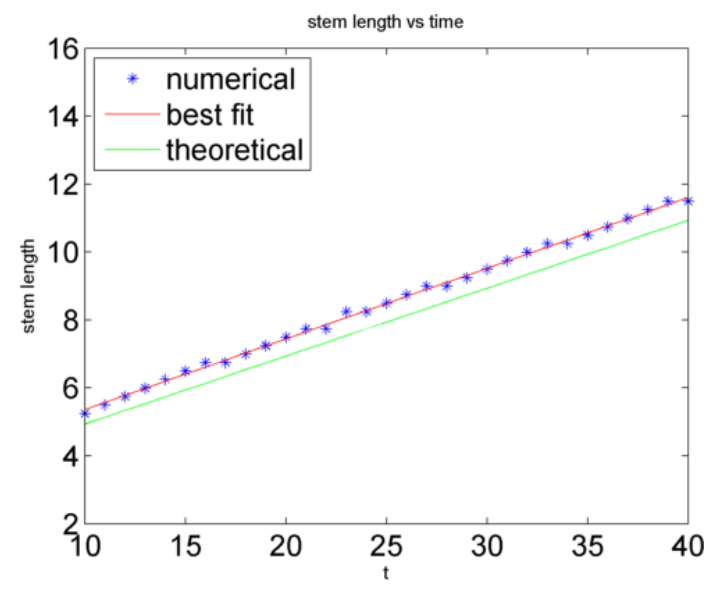

Figure 10: Growth of stem length for <-shape initial data

\subsection{Bow-shape initial data}

In this subsection, we numerically investigate the dynamics of a stem (intermediate line-soliton) in greater detail. For that purpose, we consider an initial condition obtained by adding a small, vertical stem to the $<$-shape, as shown in the top left frame of Figure 11. We call it the bow-shape initial data. Given a set of $k$-parameters $\left\{k_{1}, k_{2}, k_{3}, k_{4}\right\}$, we find that there are two distinct cases corresponding to whether the stem grows or shrinks. These cases are discussed below.

Case 1: Here we choose the bow-shape initial data that is symmetric about the $y$-axis and consists of $\mathrm{a}[1,2]$-soliton for $y \gg 0$, a $[3,4]$-soliton for $y \ll 0$, and a $[1,4]$-soliton as the initial stem. In other words, we modify the <-shape initial data for the O-type in Section 4.2 by inserting a $[1,4]$-stem in the middle. Specifically, we take

$$
u(x, y, 0)=\left\{\begin{array}{lc}
\frac{1}{2}\left(k_{2}-k_{1}\right)^{2} \operatorname{sech}^{2} \frac{1}{2}\left(k_{2}-k_{1}\right)\left[x+\left(k_{1}+k_{2}\right) y-2\left(k_{1}+k_{2}\right)\right], & y \geq 2, \\
\frac{1}{2}\left(k_{4}-k_{1}\right)^{2} \operatorname{sech}^{2} \frac{1}{2}\left(k_{4}-k_{1}\right)\left[x+\left(k_{1}+k_{4}\right) y\right], & -2 \leq y \leq 2, \\
\frac{1}{2}\left(k_{4}-k_{3}\right)^{2} \operatorname{sech}^{2} \frac{1}{2}\left(k_{4}-k_{3}\right)\left[x+\left(k_{3}+k_{4}\right) y+2\left(k_{3}+k_{4}\right)\right], & y \leq-2,
\end{array}\right.
$$

with $\left\{k_{1}, k_{2}, k_{3}, k_{4}\right\}=\{-1.1,-0.1,0.1,1.1\}$, which are the same as in Section 4.2. Note that the initial stem length is taken to be 4 units. The initial data and its time evolution are shown in the top panel of Figure 11. We observe that the stem in the initial bow shape shrinks with time by shedding energy in the form of dispersive radiation, which separates from the solitary waves. The shrinking of the stem can be explained by the KP theory in the following fashion. The top end of the stem is the intersection point of the $[1,2]$ - and the $[1,4]$-solitons which are localized along the lines

$$
L_{12}: x+\left(k_{1}+k_{2}\right) y=c_{12} t+x_{12} \quad \text { and } \quad L_{14}: x+\left(k_{1}+k_{4}\right) y=c_{14} t+x_{14} .
$$

Solving the above equations simultaneously, one obtains the coordinates $\left(x_{1}(t), y_{1}(t)\right)$ of the intersection point. In particular, $y_{1}(t)=\left(k_{1}+k_{2}+k_{4}\right) t+\left(x_{14}-x_{12}\right) /\left(k_{4}-k_{2}\right)$. Then, from the values of the $k$ parameters chosen above, $d y_{1} / d t=k_{1}+k_{2}+k_{4}<0$, implying the top end of the stem moves toward the $x$-axis. An analogous argument shows that the bottom end of the stem, which is the intersection point $\left(x_{2}(t), y_{2}(t)\right)$ of the $[3,4]$ - and the [1,4]-solitons, also moves towards the $x$-axis as $y_{2}(t)=\left(k_{1}+k_{3}+k_{4}\right) t+$ $\left(x_{34}-x_{14}\right) /\left(k_{3}-k_{1}\right)$, so that $d y_{2} / d t>0$. Consequently, the length of the stem decreases.

The resulting wave pattern evolves to yet another exact (2,2)-soliton solution of KP, namely the 2413soliton, which we do not discuss in this paper. Instead, we focus on the wave pattern formed locally around the stem. Our simulation shows that as the stem shrinks, another pair of [1,2]- and [3,4]-solitons 

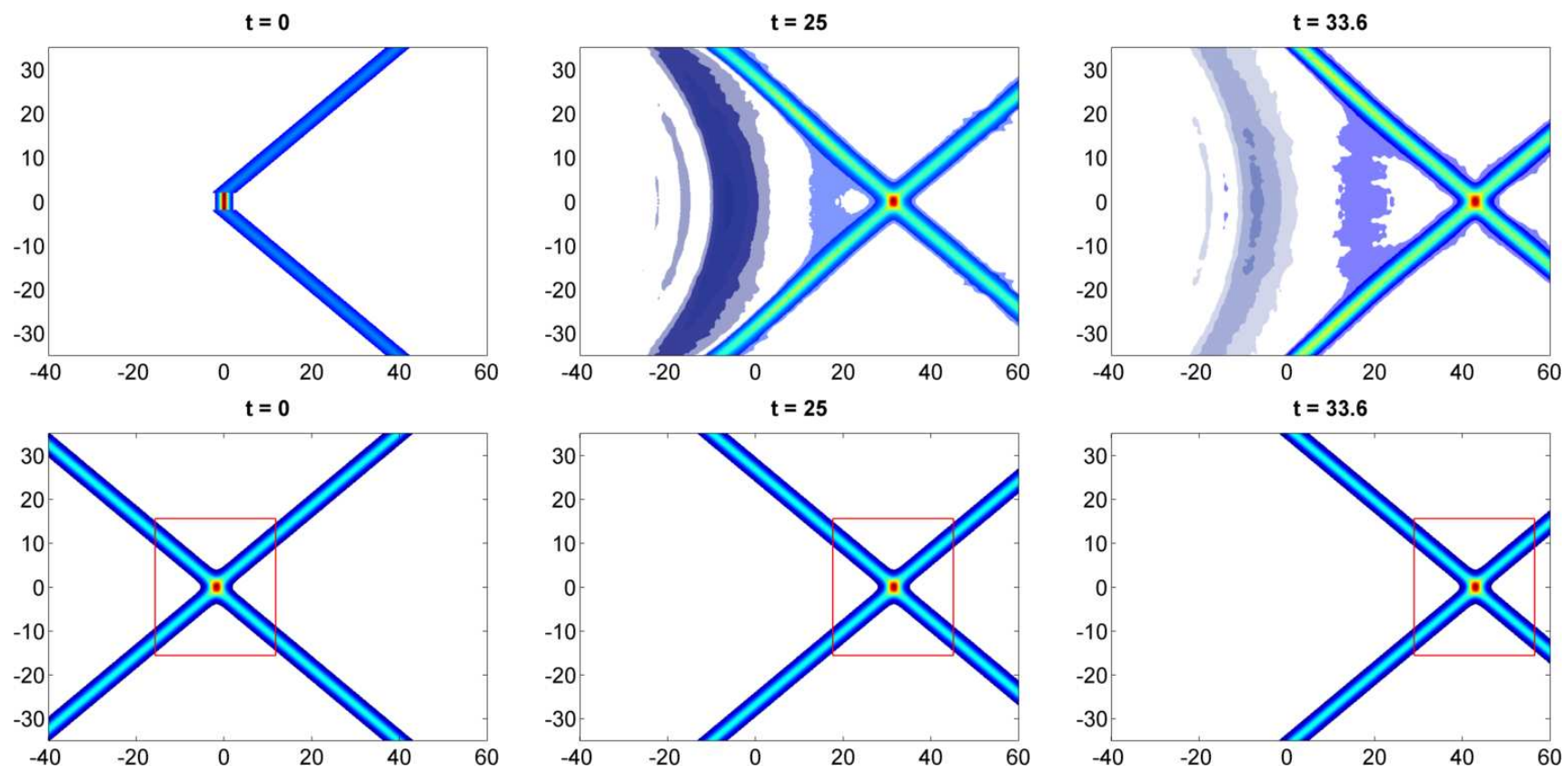

Figure 11: Evolution of a bow-shape initial wave. Initial stem length is 4 units.

are formed for $x<0$, and eventually a local O-type wave pattern (X-shape) is created. This pattern is shown in the last two frames of the top panel in Figure 11. We confirm our observation by comparing the numerical solution with an exact O-type solution with optimal parameter values which minimize the error, $E(t)$, as in Section 4.2. The exact O-type solution is shown in the bottom panel of Figure 11. We take a box size of $27.5 \times 31$, enclosing the interaction region near the vertex of the $\mathrm{X}$-shape for the minimization problem. The optimal values of the parameters for the exact solution are $(a, b)=(18.918,1.72)$ (cf. Section 4.2). The error plot on the left frame of Figure 12 shows convergence of the local wave pattern in the vicinity of the stem to an exact O-type soliton. Note that we needed to extend our numerical domain in $x$ to $[-200,200]$ for this simulation, since the local X-shape pattern moves close to the right hand boundary for run times close to $t=40$. The right frame in Figure 12 shows the evolution of the peak amplitude of
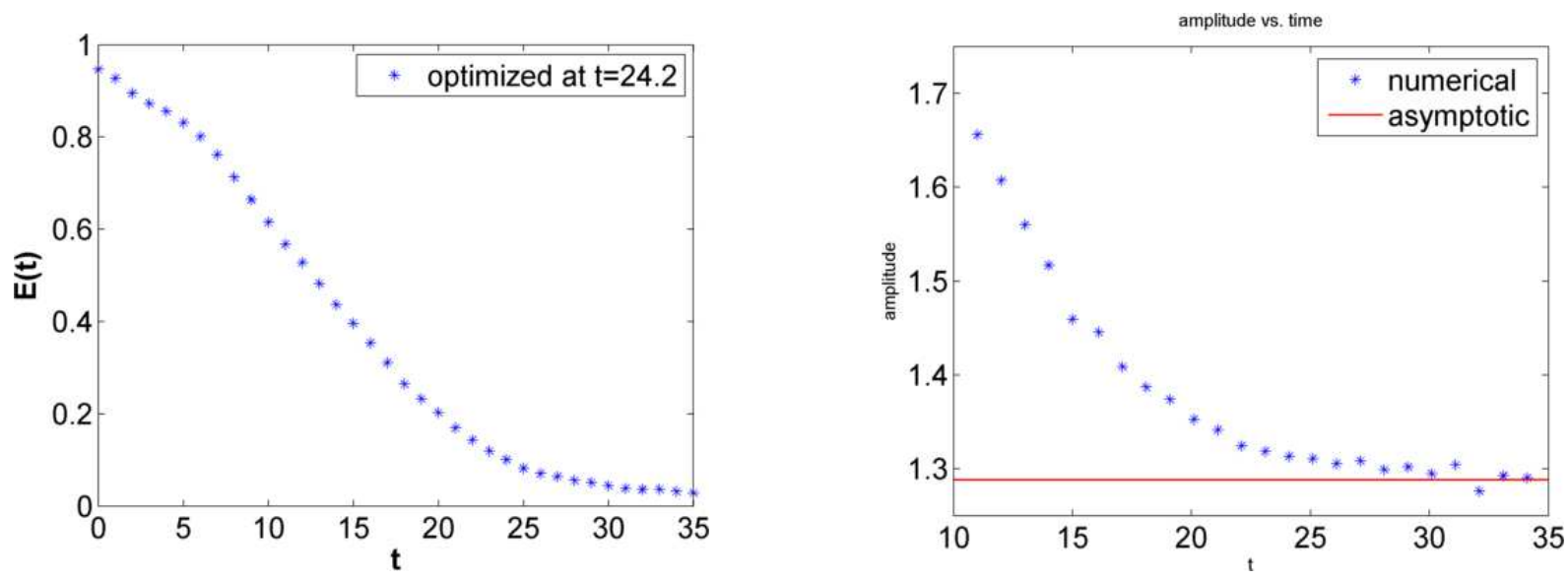

Figure 12: Convergence of a bow-shape initial data to a local O-type soliton.

the stem at $y=0$. As the stem shrinks, the peak amplitude decreases and converges to the theoretically predicted value of the maximum amplitude for the exact O-type soliton at its X-vertex. This is further evidence for the convergence of the local wave form to a O-type KP soliton. The theoretical estimate for 
the maximum amplitude at the $\mathrm{X}$-vertex of the O-type soliton is given by [5]

$$
u_{\max }=A_{1}+A_{2}+2 \sqrt{A_{1} A_{2}}\left(\frac{1-\sqrt{\Delta}}{1+\sqrt{\Delta}}\right), \quad \Delta=\frac{\left(k_{3}-k_{2}\right)\left(k_{4}-k_{1}\right)}{\left(k_{4}-k_{2}\right)\left(k_{3}-k_{1}\right)},
$$

where $A_{1}=\frac{1}{2}\left(k_{2}-k_{1}\right)^{2}, A_{2}=\frac{1}{2}\left(k_{4}-k_{3}\right)^{2}$ are the amplitudes of the $[1,2]$ and $[3,4]$ solitons, respectively. Using the chosen $k$-parameter values, we obtain $u_{\max }=1.288$, which is what our numerically estimated value approaches asymptotically in time.
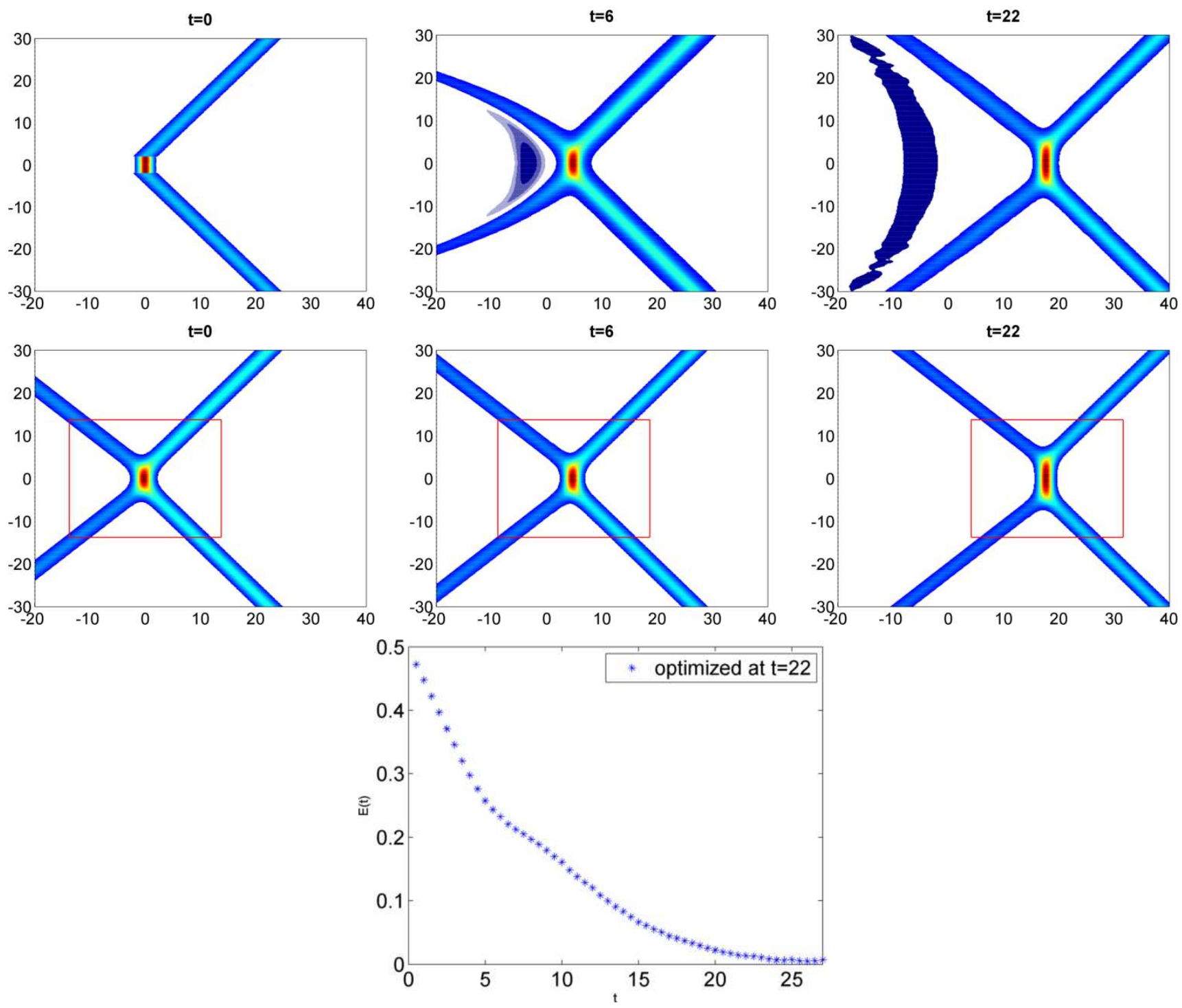

Figure 13: Evolution of a bow-shape initial wave to a 3142-soliton. Initial stem length is 4 units.

Case 2: Next we consider a bow-shape initial data

$$
u(x, y, 0)=\left\{\begin{array}{lc}
\frac{1}{2}\left(k_{3}-k_{1}\right)^{2} \operatorname{sech}^{2} \frac{1}{2}\left(k_{3}-k_{1}\right)\left[x+\left(k_{1}+k_{3}\right) y-2\left(k_{1}+k_{3}\right)\right], & y \geq 2, \\
\frac{1}{2}\left(k_{4}-k_{1}\right)^{2} \operatorname{sech}^{2} \frac{1}{2}\left(k_{4}-k_{1}\right)\left[x+\left(k_{1}+k_{4}\right) y\right], & -2 \leq y \leq 2, \\
\frac{1}{2}\left(k_{4}-k_{2}\right)^{2} \operatorname{sech}^{2} \frac{1}{2}\left(k_{4}-k_{2}\right)\left[x+\left(k_{2}+k_{4}\right) y+2\left(k_{2}+k_{4}\right)\right], & y \leq-2,
\end{array}\right.
$$

which consists of a $[1,3]$-soliton for $y \gg 0$, a [2,4]-soliton for $y \ll 0$, and a [1,4]-soliton as the initial stem of length 4 units (same as Case 1). The $k$-parameters $\left\{k_{1}, k_{2}, k_{3}, k_{4}\right\}=\{-0.9,-0.1,0.1,0.9\}$ are the same 
as in the case of the <-shape initial data in Section 4.3. The initial data and its time evolution are shown in the top panel of Figure 13. By comparing the frames at $t=6$ and $t=22$, it is clear that the stem of the bow-shape grows in this case. Furthermore, one can verify by measuring the amplitudes and slopes of the line-solitons that the initial wave form converges to a (3142)-soliton after separating from the dispersive waves. The error, $E(t)$, between the numerical and the exact 3142-solution (displayed in the second panel) is shown at the bottom of Figure 13. The error was minimized at $t=22$ by optimizing the parameters $x_{14}, x_{13}$, and $x_{24}$ corresponding to the exact 3142-soliton solution as in Section 4.3. From these optimized values of the $x_{i j}$, the parameters in the $A$-matrix are calculated to be $(a, b, c)=(1.513,1.898,0.168)$. The box size used for the minimization of the error was $27.5 \times 27.5$. The error decreases for $t>22$, showing convergence to the 3142 -soliton solution.

Behavior of the stem: In our simulations, we observe that as the stem grows, its shape and amplitude fluctuate considerably due to interaction with dispersive waves. This behavior contrasts the smooth evolution of the stem for the 3142-soliton corresponding to the <-shape initial data discussed earlier in Section 4.3. Therefore, instead of computing the stem amplitude only at $y=0$ (as in Section 4.3), here we compute the average amplitude over a region around the center $(y=0)$ of the stem and compare with the theoretical value. Figure 14 shows our results. The left frame (top panel) is a plot of the average stem amplitude with time. It shows that the stem amplitude grows asymptotically (from below) to the theoretical value. The average stem amplitude at $t=40$ is approximately 1.59 while the theoretical value is 1.62 . The right
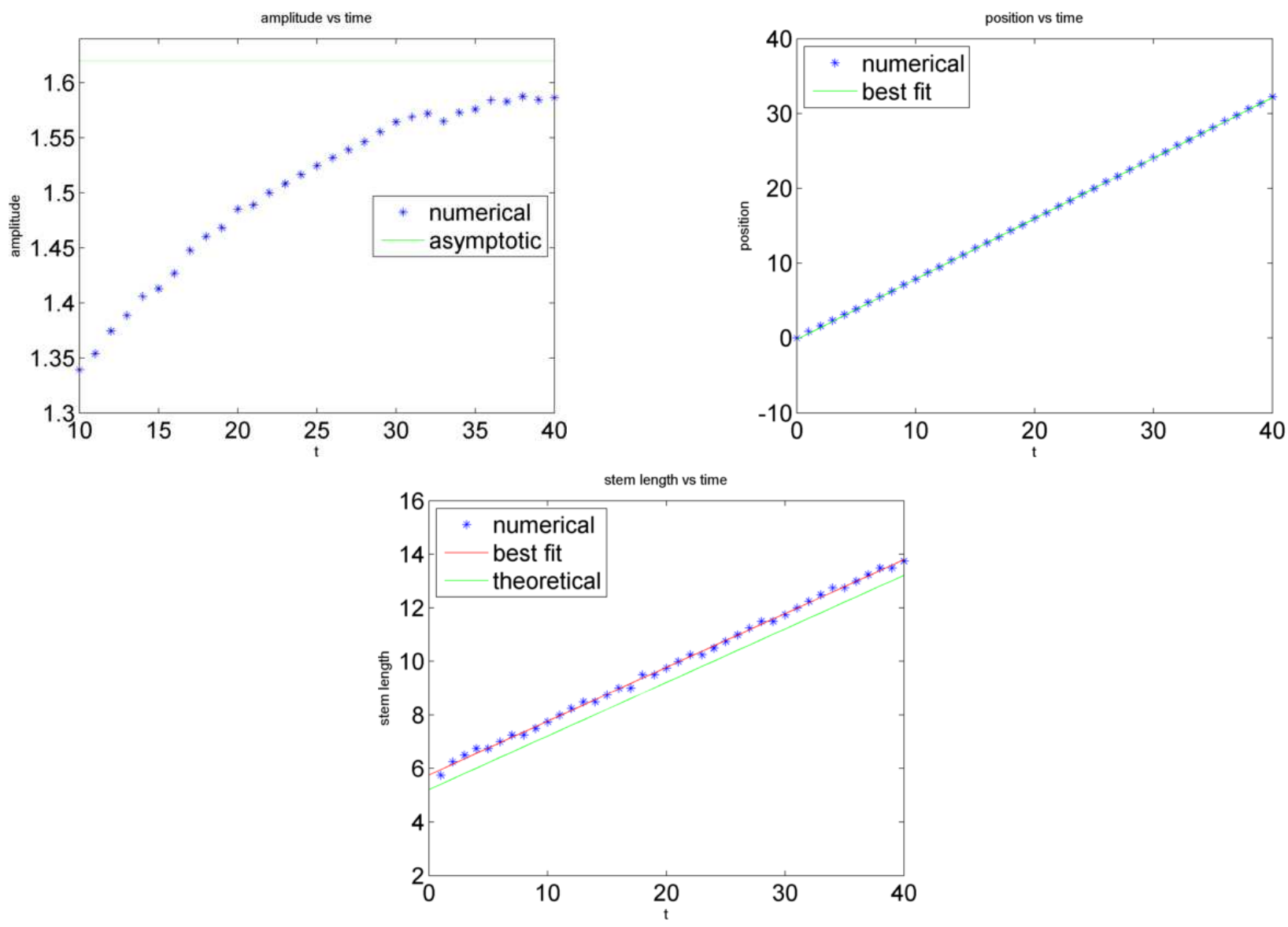

Figure 14: Stem dynamics for a bow-shape initial data

frame shows the evolution of the center of the stem at $y=0$. The slope $(=0.808)$ of the best fit line to this curve agrees with the theoretical value of the stem velocity $c_{14}=k_{1}^{2}+k_{1} k_{4}+k_{4}^{2}=0.81$.

The evolution of the stem length can be estimated from theoretical considerations in a very similar 
way as described in Case 1. For the 3142-soliton, the top and bottom ends of the stem are given by the intersection of the $[1,4]$-soliton with the $[1,3]$-soliton and the [2,4]-soliton, respectively. Carrying out a similar calculation as in Case 1, one finds that

$$
\Delta y(t)=y_{1}(t)-y_{2}(t)=\left(k_{3}-k_{2}\right) t+\frac{x_{14}-x_{13}}{k_{4}-k_{3}}+\frac{x_{14}-x_{24}}{k_{2}-k_{1}},
$$

where the $y$-coordinates of the top and bottom ends of the stem are denoted by $y_{1}(t)$ and $y_{2}(t)$, respectively. The bottom panel of Figure 14 shows the plot of the numerically estimated stem length versus time. This growth is almost linear as predicted by KP theory; the slope of the theoretical line is $2 k_{3}=0.2$, while the slope of the best fit line through the numerical data is 0.201 . The numerically estimated values for the actual stem length are slightly higher than the theoretical values for all $t$. We believe that this discrepancy (see also Figure 10) is mainly due to the method used to numerically estimate the stem length. We are trying to improve our current method.

\section{Conclusion}

In this paper we have numerically studied the initial value problem of the KP equation using a variety of initial data consisting of line solitons that are non-decaying and localized along specified rays in the $x y$-plane. Our numerical simulations demonstrate that the initial solitary waves interact nonlinearly, shedding dispersive waves and evolve to wave patterns which converge to certain exact line-soliton solutions of the KP equation. These exact solutions were classified in earlier works. Here, the convergence of the numerical solution to the exact one is only in a local sense. That is, we measure the $L^{2}$ error between the numerical and exact solution over a compact region in the $x y$-plane. It is then shown that there exist an optimal choice of parameters determining an exact KP soliton solution such that the computed error decreases with time. This implies that as the numerical wave pattern evolves, it gets closer (asymptotically in time) to the exact solution over the compact region which captures the nonlinear interaction. In our examples, we have also analyzed the nonlinear interactions which often result in the formation of intermediate line-solitons (or stems). We have studied the dynamics of the stem solitons and compared our numerical results to the KP theory. These comparisons provide further evidence that at least, to leading order, the numerically evolved wave patterns can be locally approximated by the KP line-soliton solutions.

In this work, we have only considered forms of initial data which evolve to solutions of KP containing a small number of asymptotic line solitons. In future work we plan to study more complex wave patterns containing a large number of asymptotic line-solitons. We also propose to identify the types of initial data that converge to a specific KP line-soliton solution. In particular, we are currently working to build a catalog of all initial data which evolve to the distinct types of $(2,2)$-solitons of KP. The notion of the minimal completion of the chord diagrams associated with fixed-point free permutations play a crucial role in our investigations since all line-soliton solutions have been classified in terms of these permutations. We will report these findings in a future publication.

\section{Acknowledgments}

This research was partially supported by NSF under grant numbers DMS-1108694 \& DMS-1410862. The authors thank Y. Kodama for helpful discussions and Y. Jia for a preliminary version of MATLAB code. 


\section{References}

[1] M. J. Ablowitz and H. Segur, Solitons and the inverse scattering transform, (SIAM, Philadelphia, 1981).

[2] B. B. Kadomtsev and V. I. Petviashvili, On the stability of solitary waves in weakly dispersing media, Sov. Phys. Doklady 15, 539-541 (1970)

[3] M. J. Ablowitz and D. E. Baldwin, Nonlinear shallow ocean-wave soliton interactions on flat beaches, Phys. Rev. E 86 (2012) 036305

[4] S. Chakravarty and Y. Kodama, Classification of the line-solitons of KPII, J. Phys. A: Math. Theor., 41 (2008) 275209 (33pp).

[5] S. Chakravarty and Y. Kodama, Soliton solutions of the KP equation and application to shallow water waves, Stud. Appl. Math., 123 (2009) 83-151.

[6] Y. Kodama, Young diagrams and $N$-soliton solutions of the KP equation, J. Phys. A: Math. Gen., 37 (2004) 11169-90.

[7] Y. Kodama, KP solitons in shallow water, J. Phys. A: Math. Theor, 43 (2010) 434004 (54pp).

[8] C-Y Kao and Y. Kodama, Numerical study of the KP equation for non-periodic waves, Math. Comput. Simul. 82 (2012) 1185-1218.

[9] Y. Kodama and L. Williams, KP solitons and total positivity for the Grassmannian, Invent. Math 195 (2014)

[10] Y. Kodama and H. Yeh, Two-dimensional solitons in shallow water, submitted to J. Fluid. Mech.

[11] M. Tanaka, Mach reflection of a large-amplitude solitary waves, J. Fluid Mech., 248 (1993) 637661.

[12] Y. Jia, Numerical study of the KP solitons and higher order Miles theory of the Mach reflection in shallow water, Ph.D. Thesis, Ohio State University (2014).

[13] H. Yeh, Laboratory Realization of Kodama's KP-Solitons, Lecture delivered at the NSF/CBMS Regional Conference in the Mathematical Sciences "Solitons in Two-Dimensional Water Waves and Applications to Tsunami", UTPA, May 20-24, 2013 (Lecture slides are available at http://faculty.utpa.edu/kmaruno/nsfcbms-tsunami.html). 